Pacific Journal of Mathematic 


\section{DIFFERENTIABILITY CONDITIONS AND BOUNDS ON SINGULAR POINTS}

\section{GARY SPOAR}

It is well-known that a normal arc $\mathscr{A}_{4}$ of cyclic order four in the conformal plane contains at most finitely many singular points and in fact at most eleven. This bound can be reduced to four in the case of a strongly differentiable $\mathscr{A}_{4}$. Using a characterization of singular points on such arcs this paper shows that strong differentiability is not a necessary condition for this bound. In fact a much weaker condition, viz., the existence of tangent circles, is sufficient to obtain four as the least upper bound.

In [4] a conformal proof is given for the following result. "A normal arc $\mathscr{A}_{4}$ of cyclic order four contains at most eleven singular points."

That a strongly differentiable ([3], 3.1) $\mathscr{A}_{4}$ contains at most four singular points can be found in 4.1.4.3 of [1] and in 3.6 of [4].

In $\S 3$ it is shown that assuming only Condition I $([3], 1.5)$ the maximum number of singular points on such arcs and curves is still four and that this is the best possible bound.

In order to obtain this result it is necessary to categorize the different possible types of singular points on such arcs. This characterization is similar to that of $\mathrm{O}$. Haupt and $\mathrm{H}$. Künneth for linearly singular points of arcs of linear order three ([1], 3.2.1).

The definitions and notations used in this paper can be found in [2] and [3]. We include the notations of ordinary and strong conformal differentiability for the reader's convenience. It is obvious that strong differentiability implies ordinary differentiability.

(a) A point $p$ on an arc $\mathscr{A}$ is said to be (conformally) differentiable if it satisfies two conditions:

Condition I. For every point $R \rightleftharpoons p$, and for every sequence of points $s \rightarrow p, s \in A, s \neq p$, there exists a circle $C_{0}$ such that $C(p, s$, $R) \rightarrow C_{0} . \quad C_{0}$ is called the tangent circle of $\mathscr{A}$ at $p$ through $R$ and is denoted $C(\tau, R)$.

Condition II. If $s \rightarrow p, s \nsim p$, there exists a circle $C(p)$ such that $C(\tau, s) \rightarrow C(p) . \quad C(p)$ is called the osculating circle of $\mathscr{A}$ at $p$.

(b) A point $p$ on an arc $\mathscr{A}$ is said to be (conformally) strongly differentiable if it satisfies the following conditions: 
Condition I'. Let $R \rightleftharpoons p, Q \rightarrow R$. If two distinct points $u$ and $v$ converge on $\mathscr{A}$ to $p$, then $C(u, v, Q)$ always converges.

Condition $\mathrm{II}^{\prime} . \quad C(t, u, v)$ converges if the three mutually distinct points $t, u, v$ converge on $\mathscr{A}$ to $p$.

1. Types of singular points. Hereafter, when considering a two-sided neighbourhood $L \cup\{z\} \cup M$ of an interior point $z$ on an arc $\mathscr{A}$, we assume that $\mathscr{A}$ has been oriented so that $L[M]$ is a preceding [proceeding] one-sided neighbourhood of $z$ on $\mathscr{A}$.

DEFINITION. Let $z$ be a singular point of an oriented arc $\mathscr{A}_{4}$ of cyclic order four. Then $z$ is $(1,3)[(3,1) ;(2,2)]$ singular provided that for any two-sided neighbourhood $L \cup\{z\} \cup M$ of $z$ on $\mathscr{A}_{4}$ there exists a circle which meets $L$ and $M$ once and three times [three times and once; twice], respectively.

REMARK. (i) Counting multiplicities for this definition, each of the points in common with the circle and $\mathscr{A}_{4}$ must be intersection points.

(ii) For normal ares $\mathscr{A}_{4}$ of cyclic order four, these are the only possible singular points. Otherwise, we would have what might be called a $(4,0)$ or $(0,4)$ singular point $z$. But $z$ is elementary ([3], 5.1) since $\mathscr{A}_{4}$ contains only finitely many singular points. Thus a $(4,0)$ or $(0,4)$ singular point does not exist for normal arcs $\mathscr{A}_{4}$ of cyclic order four.

(iii) In each case there exists a limiting supporting general osculating circle of $\mathscr{A}_{4}$ at $z$ which may be the point circle. If $z$ is $(1,3)$ or $(3,1)$ singular, then one of the limiting supporting general osculating circles is the one-sided osculating circle of $\mathscr{A}_{4}$ at $z$ from the right or left, respectively, since $\mathscr{A}_{4}$ is one-sidedly strongly differentiable at $z$; cf. ([4], 3.4).

(iv) Let $\mathscr{A}_{4}$ be differentiable at a singular point $z$. Then the osculating circle supports $\mathscr{A}_{4}$ at $z$; cf. ([2], 9) and ([3], Theorem 4). But $\mathscr{A}_{4}$ is one-sidedly strongly differentiable at $z$. Thus differentiable singular points are both $(3,1)$ and $(1,3)$ singular.

For the remainder of the paper $\mathscr{A}_{4}$ will denote a normal oriented arc of cyclic order four. Since end-points of such arcs are ordinary; cf. ([4], 3.3), we will restrict our attention to open arcs. The inclusion of end points will involve no additional singular points. 
LEMMA 1.1. Let $z$ be a $(2,2)$ singular point on $\mathscr{A}_{4}$ which is neither $(3,1)$ nor $(1,3)$ singular. Then $\mathscr{A}_{4}$ does not satisfy Condition I at $z$.

Proof. Since $z$ is neither $(3,1)$ nor $(1,3)$ singular, the two onesided osculating circles of $\mathscr{A}_{4}$ at $z$ intersect $\mathscr{A}_{4}$ at $z$. Let us assume that $\mathscr{A}_{4}$ satisfies Condition $I$ at $z$. Thus the family $\tau$ of tangent circles of $\mathscr{A}_{4}$ at $z$ is a pencil of the second kind at $z$; i.e., the circles of $\tau$ touch each other at $z$. There are two possible cases to consider.

Case (i). $z$ is not a cusp point; i.e., the nontangent circles of $\mathscr{A}_{4}$ at $z$ all intersect $\mathscr{A}_{4}$ at $z$. But then $\mathscr{A}_{4}$ satisfies Condition I' ([3], 3.1) at $z$; cf. ([3], Theorem 5). Let $L \cup\{z\} \cup M$ be a sufficiently small two-sided neighbourhood of $z$ on $\mathscr{A}$. Since $z$ is $(2,2)$ singular, but not $(3,1)$ or $(1,3)$ singular, there is a circle which intersects each of $L$ and $M$ at two points but no circle which meets $L$ three times and $M$ once, or $L$ once and $M$ three times. Call these points $l<l^{\prime}<$ $m^{\prime}<m$.

Now let $t$ move monotonically and continuously from $l^{\prime}$ on $\mathscr{A}_{4}$ toward $z$. Then there is a point $u \in C(l, t, m)$ on $\mathscr{A}_{4}$ which moves monotonically and continuously from $m^{\prime}$ toward $z$; cf. ([4], 2.1). This movement will yield $t=u=z$ as $t$ reaches $z$. Thus we obtain a general tangent circle $K$ of $\mathscr{A}_{4}$ at $z$ intersecting $L$ at $l$ and $M$ at $m$. But $K$ is then a tangent circle of $\mathscr{A}_{4}$ at $z$ belonging to the pencil $\tau$ above, since Condition $I^{\prime}$ is satisfied at $z$. Also $K$ supports $\mathscr{A}_{4}$ at $z$; otherwise a suitable circle close to $K$ would intersect $\mathscr{A}_{4}$ at three points close to $z$ and also at $l$ and $m$, thus violating the order of $\mathscr{A}_{4}$. But then, since Condition $I^{\prime}$ is satisfied at $z$, a circle close to $K$ through $l, r, r^{\prime}$, where $r, r^{\prime} \in L$ with $r, r^{\prime}$ close to $z$, will meet $M$ at a point $s$ close to $m$. Thus this circle meets $L$ three times and $M$ once; a contradiction.

Case (ii). $z$ is a cusp point; i.e., the nontangent circles of $\mathscr{A}_{4}$ at $z$ all support $\mathscr{A}_{4}$ at $z$. Let $L \cup\{z\} \cup M$ be a small two-sided neighbourhood of $z$ on $\mathscr{A}_{4}$. Let $r \in L$. Then the tangent circle $C(\tau, r)$ of $\mathscr{A}_{4}$ at $z$ through $r$ is close to the osculating circle $C$ of $L \cup\{z\}$ at $z$. Since $C$ intersects $\mathscr{A}_{4}$ at $z$, then $C(\tau, r)$ supports $\mathscr{A}_{4}$ at $z$. Next let $s$ be close to $z$ on $L, s \neq r$. Then the nontangent circle $C(z, s, r)$ is close to $C(\tau, r)$ and supports $\mathscr{A}_{4}$ at $z$, since $z$ is a cusp point. It therefore intersects $M$ at a point $t$. Hence a circle close to $C(z, s, r)$ will meet $\mathscr{A}_{4}$ at least five times; a contradiction.

Thus our assumption that $\mathscr{A}_{4}$ satisfies Condition I is incorrect and we have desired result. 


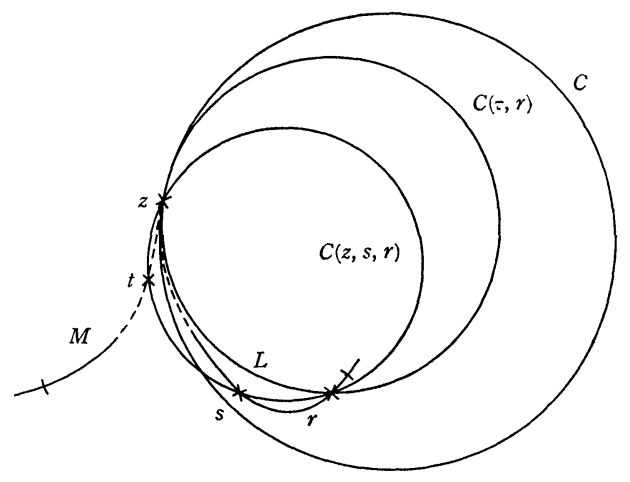

2. $\Gamma_{a}$-singular points. Let $a$ be the end-point of $\mathscr{A}_{4}$ with $a<s$ for all $s \in \mathscr{A}_{4}$. Let $\Gamma_{a}$ be the system of circles passing through $a$. Then $y$ is $\Gamma_{a}$-singular ([4], 2.3) if, for any neighbourhood $N$ of $y$ on $\mathscr{A}_{4}$ there exists a circle of $\Gamma_{a}$ that meets $N$ at least three times.

Lemma 2.1. Let $z_{1}, z_{2}$ be two singular points of $\mathscr{A}_{4}$. Also let $a$ be the end-point of $\mathscr{A}_{4}$ with $a<z_{1}<z_{2}$. Then

(a) If $z_{1}$ and $z_{2}$ are $(3,1)$ and $(1,3)$ singular, respectively, then there is at least one $\Gamma_{a}$-singular point $s$ on $\mathscr{A}_{4}$ with $z_{1} \leqq s \leqq z_{2}$.

(b) If $z_{1}$ and $z_{2}$ are $(1,3)$ and $(3,1)$ singular, respectively, then there is at least one $\Gamma_{a}$-singular point $s$ on $\mathscr{A}_{4}$ with $z_{1}<s<z_{2}$.

(c) If $z_{1}, z_{2}$ are both $(1,3)$ singular, then there is at least one $\Gamma_{a}$-singular point $s$ on $\mathscr{A}_{4}$ with $z_{1}<s \leqq z_{2}$.

(d) If $z_{1}, z_{2}$ are both $(3,1)$ singular, then there is at least one $\Gamma_{a}$-singular point $s$ on $\mathscr{A}_{4}$ with $z_{1} \leqq s<z_{2}$.

Proof. In all cases there exists at least one $\Gamma_{a}$-singular point $s$ on $\mathscr{A}_{4}$ with $z_{1} \leqq s \leqq z_{2}$; cf. ([4], 2.5). Hence (a) is obtained automatically.

To obtain (b) refer to the proof of 2.4 in [4], where $N_{1}=L_{1} \cup$ $\left\{z_{1}\right\} \cup M_{1}$ and $N_{2}=L_{2} \cup\left\{z_{2}\right\} \cup M_{2}$ are two-sided neighbourhoods of $z_{1}$ and $z_{2}$ on $\mathscr{A}_{4}$, respectively. Since $z_{1}$ is $(1,3)$ singular there exists a circle meeting $L_{1}$ once and $M_{1}$ three times. Now $z_{2}$ is $(3,1)$ singular. Hence there exists a circle meeting $L_{2}$ three times and $M_{2}$ once. The process used in the proof with respect to these two pairs of quadruplets enables one to construct a circle of $\Gamma_{a}$ meeting the closed subarc $\mathscr{B}$ of $\mathscr{A}_{4}$ bounded by $z_{1}$ and $z_{2}$ at three interior points. Then by 2.3 of [4], there exists at least one $\Gamma_{a}$-singular point $s$ in the interior of $\mathscr{B}$; i.e., $z_{1}<s<z_{2}$. Arguments similar to those used above yield (c) and (d). 
LEMmA 2.2. Let $z_{1}<z_{2}<\cdots<z_{n}$ be $n$ singular points of $\mathscr{A}_{4}$ none of which are $(2,2)$ singular. Let $a$ be the end-point of $\mathscr{A}_{4}$ with $a<z_{1}$. Then $\mathscr{A}_{4}$ contains at least $n-1 \Gamma_{a}$-singular points.

Proof. By our assumptions and remark (ii) of $\S 1, z_{i}$ is $(1,3)$ singular but not $(3,1)$ singular, or $(3,1)$ singular but not $(1,3)$ singular, or both $(3,1)$ and $(1,3)$ singular; $i=1,2, \cdots, n$. For $i=1,2, \cdots$, $n-1$, define the intervals

$$
\left\langle z_{i}, z_{i+1}\right\rangle=\left\{\begin{array}{l}
\left(z_{i}, z_{i+1}\right) \text { if } z_{i} \text { is }(1,3) \text { singular and } z_{i+1} \text { is }(3,1) \\
\quad \text { singular }, \\
{\left[z_{i}, z_{i+1}\right] \text { if } z_{i} \text { is not }(1,3) \text { singular and } z_{i+1} \text { is not }} \\
(3,1) \text { singular }, \\
{\left[z_{i}, z_{i+1}\right) \text { if } z_{i} \text { is not }(1,3) \text { singular and } z_{i+1} \text { is }(3,1)} \\
\quad \text { singular }, \\
\left(z_{i}, z_{i+1}\right] \text { if } z_{i} \text { is }(1,3) \text { singular and } z_{i+1} \text { is not }(3,1) \\
\quad \text { singular. }
\end{array}\right.
$$

Then $\left\langle z_{i}, z_{i+1}\right\rangle$ and $\left\langle z_{i+1}, z_{i+2}\right\rangle$ are disjoint subsets of $\mathscr{A}_{4}$ for $i=1,2$, $\cdots, n-2$, and by Lemma 2.1 there is a $\Gamma_{a}$-singular point in $\left\langle z_{i}, z_{i+1}\right\rangle$ for $i=1,2, \cdots, n-1$. Hence $\mathscr{A}_{4}$ contains at least $n-1 \Gamma_{a}$-singula1 points.

3. The main result. We are now ready to derive the desired result.

THEOREM 1. A normal arc $\mathscr{A}_{4}$ of cyclic order four, satisfying Condition I at each point, contains at most four singular points.

Proof. By Lemma 1.1, all the singular points of $\mathscr{A}_{4}$ are of type $(3,1)$ or $(1,3)$. Let us assume that $\mathscr{A}_{4}$ contains at least five singular points $z_{1}, z_{2}, z_{3}, z_{4}, z_{5}$. If $a$ is the end-point of $\mathscr{A}_{4}$ with $a<z_{1}$, then by Lemma $2.2 \mathscr{A}_{4}$ contains at least four $\Gamma_{a}$-singular points.

Now let $\mu$ be a Möbius transformation mapping $a$ to $\infty$. Then removing $\mu$ (a) we have the open plane. But $\Gamma_{a}$-singular points now become linearly singular points (points of linear order three). Also let $L_{1}$ be a small preceding neighbourhood of $z_{1}$ and $M$ a small proceeding neighbourhood of $z_{5}$. Then the arc $\mu\left(L_{1} \cup\left[z_{1}, z_{5}\right] \cup M_{5}\right)$ is a bounded normal arc of linear order three containing at least four linearly singular points. This is known to be impossible; cf. ([1], 3.2.4). Thus $\mathscr{A}_{4}$ contains at most four singular points.

A closed curve $C_{4}$ of cyclic order four is automatically normal. 
Using the method of the proof of Theorem 1, one obtains the following result.

THEOREM 2. A closed curve $C_{4}$ of cyclic order four satisfying Condition I contains at most four singular points.

Finally, we should note that there are normal arcs and curves of cyclic order four satisfying Condition I which contain four singular points. For example, consider any arc of the ellipse given by the equation $3 x^{2}+4 y^{2}=12$ containing the points $P_{1}(0, \sqrt{3}), P_{2}(2,0), P_{3}(0$, $-\sqrt{3})$ and $P_{4}(-2,0)$ or the ellipse itself. Each $P_{i}, i=1,2,3,4$ is a singular point of this arc.

Hence the maximum number of four singular points for normal arcs and curves of cyclic order four satisfying Condition I obtained in Theorems 1 and 2 is the best possible bound.

\section{REFERENCES}

1. O. Haupt and H. Künneth, Geometrische Ordnungen, Springer-Verlag, Berlin (1967). 2. N. D. Lane and P. Scherk, Differentiable points in the conformal plane, Canad. J. Math., 5 (1953), 512-518.

3. Characteristic and order of differentiable points in the conformal plane, Trans. Amer. Math. Soc., 81 (1956), 358-378.

4. N. D. Lane and G. Spoar, On singular points of normal arcs of cyclic order four, Canad. Math. Bull., 17 (3) (1974), 391-396.

Received January 30, 1975.

UNIVERSITY OF GUELPH 


\section{PACIFIC JOURNAL OF MATHEMATICS}

\section{EDITORS}

RICHARD ARENS (Managing Editor) University of California

Los Angeles, California 90024

\section{R. A. BEAUMONT}

University of Washington Seattle, Washington 98105
J. DugundjI Department of Mathematics University of Southern Californı Los Angeles, California 90007

D. Gilbarg AND J. Milgram Stanford University Stanford, California 94305

\section{ASSOCIATE EDITORS}

E. F. BECKENBACH

B. H. NeUMANN

F. WOLF

K. YosHidA

\section{SUPPORTING INSTITUTIONS}

UNIVERSITY OF BRITISH COLUMBIA CALIFORNIA INSTITUTE OF TECHNOLOGY UNIVERSITY OF CALIFORNIA MONTANA STATE UNIVERSITY UNIVERSITY OF NEVADA NEW MEXICO STATE UNIVERSITY OREGON STATE UNIVERSITY UNIVERSITY OF OREGON OSAKA UNIVERSITY

\author{
UNIVERSITY OF SOUTHERN CALIFORNIA \\ STANFORD UNIVERSITY \\ UNIVERSITY OF TOKYO \\ UNIVERSITY OF UTAH \\ WASHINGTON STATE UNIVERSITY \\ UNIVERSITY OF WASHINGTON \\ AMERICAN MATHEMATICAL SOCIETY \\ NAVAL WEAPONS CENTER
}




\section{Pacific Journal of Mathematics}

\section{Vol. 61, No. $1 \quad$ November, 1975}

Jiří Adámek, V. Koubek and Věra Trnková, Sums of Boolean spaces represent every

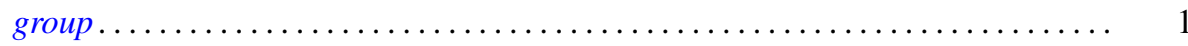

Richard Neal Ball, Full convex l-subgroups and the existence of $a^{*}$-closures of

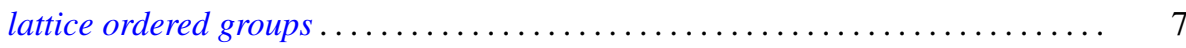

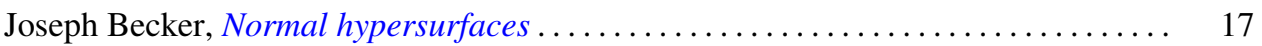

Gerald A. Beer, Starshaped sets and the Hausdorff metric . . . . . . . . . . . . . 21

Dennis Dale Berkey and Alan Cecil Lazer, Linear differential systems with

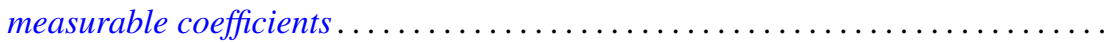

Harald Boehme, Glättungen von Abbildungen 3-dimensionaler

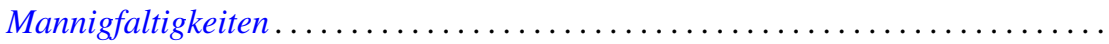

Stephen LaVern Campbell, Linear operators for which $T^{*} T$ and $T+T^{*}$

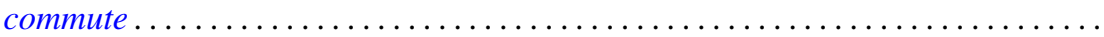

H. P. Dikshit and Arun Kumar, Absolute summability of Fourier series with

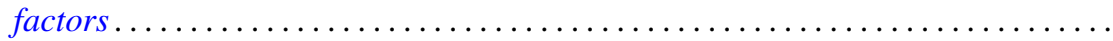

Andrew George Earnest and John Sollion Hsia, Spinor norms of local integral

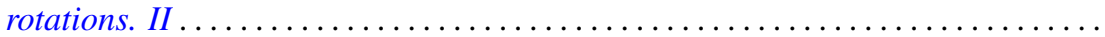

Erik Maurice Ellentuck, Semigroups, Horn sentences and isolic structures .........

Ingrid Fotino, Generalized convolution ring of arithmetic functions . . . . . . . . . . .

Michael Randy Gabel, Lower bounds on the stable range of polynomial rings .......

Fergus John Gaines, Kato-Taussky-Wielandt commutator relations and

characteristic curves

Theodore William Gamelin, The polynomial hulls of certain subsets of $C^{2}$

R. J. Gazik and Darrell Conley Kent, Coarse uniform convergence spaces. . .

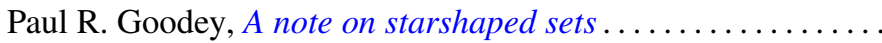

Eloise A. Hamann, On power-invariance

M. Jayachandran and M. Rajagopalan, Scattered compactification for $N \cup\{P\}$. . .

V. Karunakaran, Certain classes of regular univalent functions .

John Cronan Kieffer, A ratio limit theorem for a strongly subadditive set function in a locally compact amenable group .................

Siu Kwong Lo and Harald G. Niederreiter, Banach-Buck measure, density, and uniform distribution in rings of algebraic integers ........

Harold W. Martin, Contractibility of topological spaces onto metric spaces ....

Harold W. Martin, Local connectedness in developable spaces .

A. Meir and John W. Moon, Relations between packing and covering numbers of a tree.

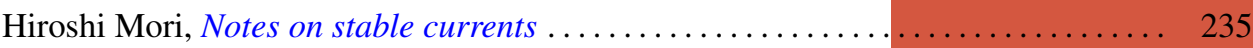

Donald J. Newman and I. J. Schoenberg, Splines and the logarithmic function . . . . 241

M. Ann Piech, Locality of the number of particles operator....

Fred Richman, The constructive theory of $K T$-modules .......

Gerard Sierksma, Carathéodory and Helly-numbers of

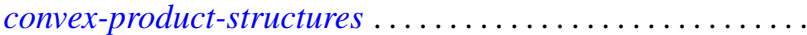

Raymond Earl Smithson, Subcontinuity for multifunctions .... . . 